\title{
Imaging Particle Analysis Using a High Resolution In-Flow Imaging Flow Cytometer with Oil Immersion Optics
}

\author{
L.H. Brown, * C.K. Sieracki* \\ * Fluid Imaging Technologies, Inc., 65 Forest Falls Drive, Yarmouth, ME 04096
}

This paper will describe a novel new method (patent pending) for imaging particles in-flow at a resolution approaching $0.30 \mu \mathrm{m}$. The system described uses oil immersion techniques to dramatically increase the resolution capabilities of a standard air immersion microscope optical system. While oil immersion microscopes are quite common, this method is unique in two crucial ways: 1.) the microscope is taking pictures of moving particles in-flow and 2.) oil immersion is used on both the microscope objective as well as the condenser optics.

Oil immersion techniques are used in microscopy to increase the resolution of the microscope by increasing the Numerical Aperture (NA) of the objective lens. Using an oil immersion fluid having an index of refraction near to that of the optics in between the objective and sample eliminates the loss of light usually occurring due to the air/glass interface, thereby increasing the overall NA and maintaining the clarity and resolving power of the objective.

In this system, the particles being observed are moving through a rectangular flow cell having a very narrow depth $(50 \mu \mathrm{m})$ perpendicular to the optical axis. The "walls" of this flow cell are extremely thin, roughly $100 \mu \mathrm{m}$. A peristaltic pump and tubing "pulls" the sample through the flow cell. Oil immersion fluid is placed between the condenser lens and one side of the flow cell, while the other side of the flow cell also has oil immersion fluid between it and a 100X oil immersion objective. A digital firewire camera collects images of the passing particles viewed through the optics. The condenser light source is pulsed in synchronization with the camera acquisition to help in "freezing" the moving particles.

One issue of this setup is that the depth of focus at this magnification is very limited, probably less than $10 \mu \mathrm{m}$. Given the depth of the flow cell $(50 \mu \mathrm{m})$, this means that a large majority of the passing particles will not be in sharp focus. In order to overcome this, an edge-gradient filter is applied to each incoming particle image, and only those particle images having a high edge gradient are kept. This insures that the particle images kept by the system are all in sharp focus. Since the sample is continuously flowing through the flow cell, one can keep acquiring particle images until a desired number are saved. This means that very large numbers of particle images can be collected and saved in a relatively short period of time, yielding far higher statistical significance for any measurements made than can be obtained using manual microscopy. Since the system can store up to 26 different measurements for each particle, automated pattern recognition algorithms can also be used in postprocessing to classify particles into different types. Due to the higher resolution of these images, the measurements are more exact, and thus image classification can be done on particles much smaller than previously capable in this type of system. Figure 1 shows an example of laser toner particles imaged both with the new oil immersion system and a standard system without the oil immersion. The results are clearly significantly better from the new system. 


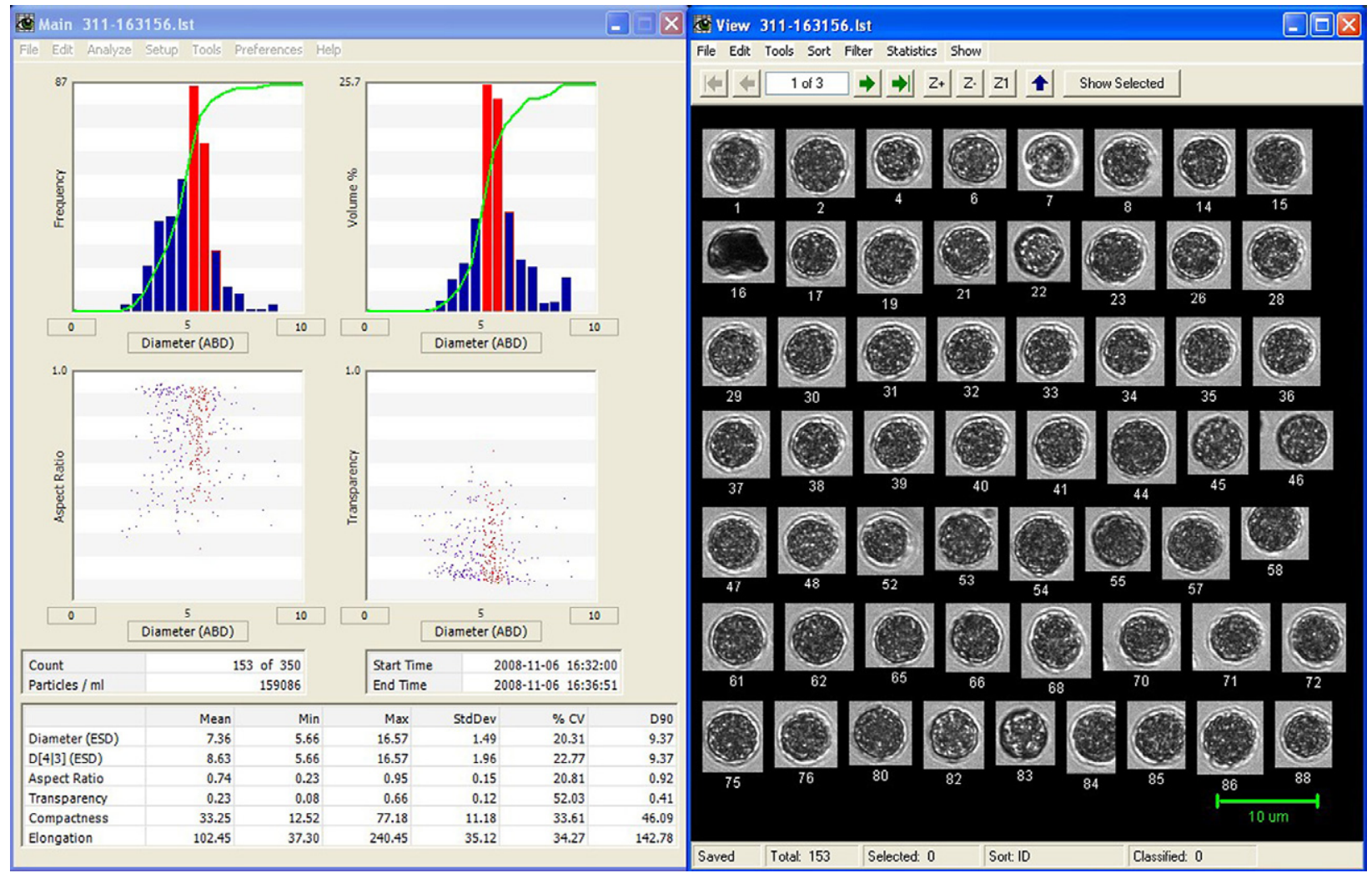

Figure 1: Laser toner particles imaged with 1.3 Numerical Aperture (NA) oil immersion system (above) and 0.5 NA air immersion (standard) system below.

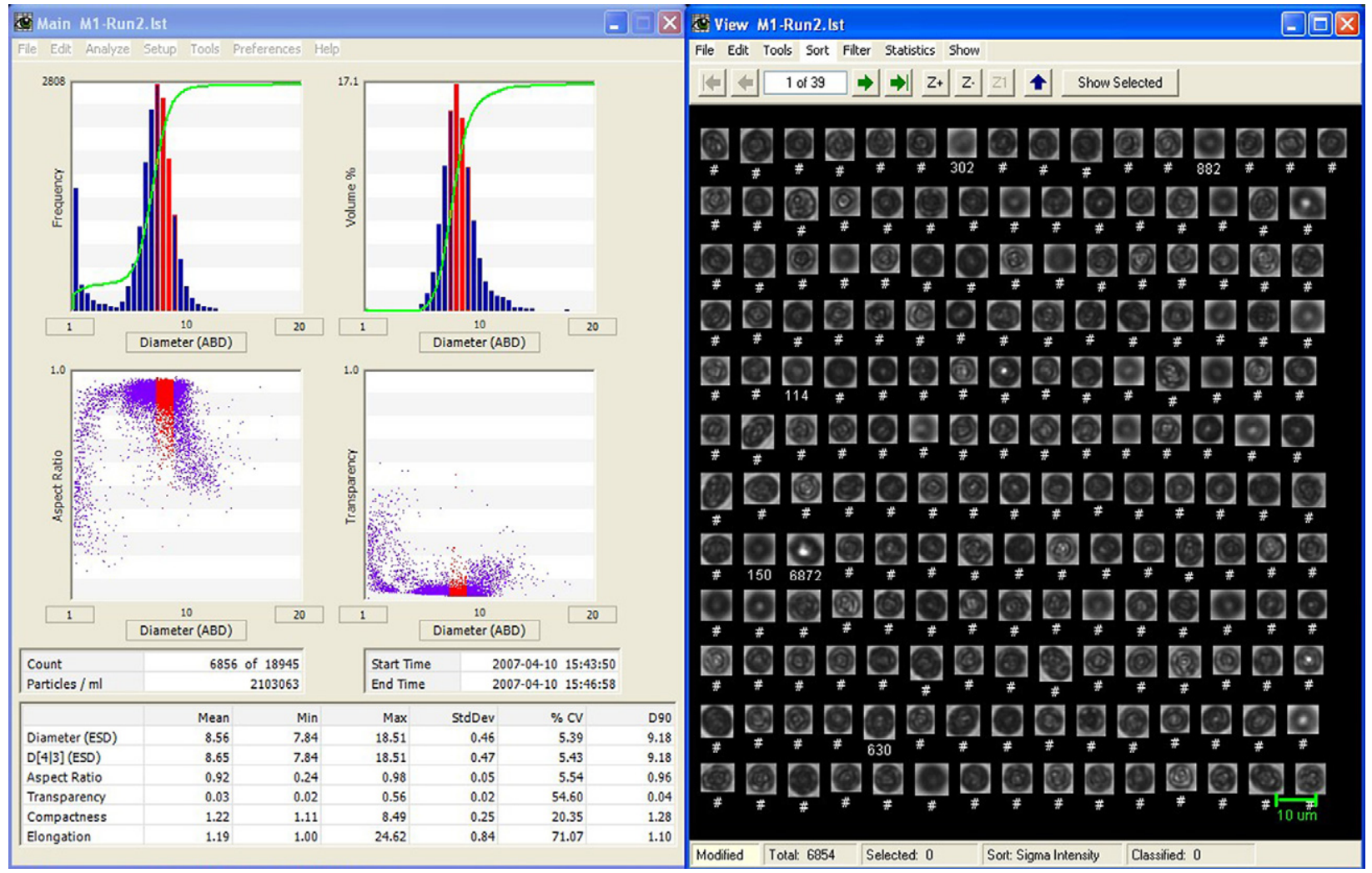

